\title{
Weighted boundedness for Toeplitz type operators related to strongly singular integral operators
}

\section{Dazhao Chen*}

\section{"Correspondence:}

chendazhao27@sina.com

Department of Science and

Information Science, Shaoyang

University, Shaoyang, Hunan

422000, P.R. China

\begin{abstract}
In this paper, we show the sharp maximal function estimates for the Toeplitz type operators related to the strongly singular integral operators. As an application, we obtain the boundedness of the operators on weighted Lebesgue and Triebel-Lizorkin spaces.

MSC: $42 \mathrm{~B} 20 ; 42 \mathrm{~B} 25$

Keywords: Toeplitz operator; strongly singular integral operator; sharp maximal function; Triebel-Lizorkin space; weighted Lipschitz function
\end{abstract}

\section{Introduction and Preliminaries}

As a development of singular integral operators [1,2], their commutators have been well studied. In [3-5], the authors prove that the commutators generated by the singular integral operators and $B M O$ functions are bounded on $L^{p}\left(R^{n}\right)$ for $1<p<\infty$. Chanillo [6] proves a similar result when singular integral operators are replaced by the fractional integral operators. In [7-9], the boundedness for the commutators generated by the singular integral operators and Lipschitz functions on Triebel-Lizorkin and $L^{p}\left(R^{n}\right)(1<p<\infty)$ spaces are obtained. In $[10,11]$, the boundedness for the commutators generated by the singular integral operators and the weighted $B M O$ and Lipschitz functions on $L^{p}\left(R^{n}\right)$ $(1<p<\infty)$ spaces are obtained. In [12,13], some Toeplitz type operators related to the singular integral operators and strongly singular integral operators are introduced, and the boundedness for the operators generated by $B M O$ and Lipschitz functions is obtained. In this paper, we will study the Toeplitz type operators related to the strongly singular integral operator and the weighted Lipschitz functions.

First, let us introduce some notation. Throughout this paper, $Q$ will denote a cube of $R^{n}$ with sides parallel to the axes. For any locally integrable function $f$, the sharp maximal function of $f$ is defined by

$$
M^{\#}(f)(x)=\sup _{Q \ni x} \frac{1}{|Q|} \int_{Q}\left|f(y)-f_{Q}\right| d y,
$$

where we write $f_{Q}=|Q|^{-1} \int_{Q} f(x) d x$. It is well known that $[1,2]$

$$
M^{\#}(f)(x) \approx \sup _{Q \ni x} \inf _{c \in C} \frac{1}{|Q|} \int_{Q}|f(y)-c| d y .
$$

O2014 Chen; licensee Springer. This is an Open Access article distributed under the terms of the Creative Commons Attribution License (http://creativecommons.org/licenses/by/2.0), which permits unrestricted use, distribution, and reproduction in any medium, provided the original work is properly cited. 
Let

$$
M(f)(x)=\sup _{Q \ni x} \frac{1}{|Q|} \int_{Q}|f(y)| d y .
$$

For $\eta>0$, set $M_{\eta}(f)(x)=M\left(|f|^{\eta}\right)^{1 / \eta}(x)$.

For $0<\eta<1$ and $1 \leq r<\infty$, set

$$
M_{\eta, r}(f)(x)=\sup _{Q \ni x}\left(\frac{1}{|Q|^{1-r \eta / n}} \int_{Q}|f(y)|^{r} d y\right)^{1 / r} .
$$

The $A_{p}$ weight is defined by [1]

$$
\begin{aligned}
A_{p} & =\left\{w \in L_{\mathrm{loc}}^{1}\left(R^{n}\right): \sup _{Q}\left(\frac{1}{|Q|} \int_{Q} w(x) d x\right)\left(\frac{1}{|Q|} \int_{Q} w(x)^{-1 /(p-1)} d x\right)^{p-1}<\infty\right\}, \\
& 1<p<\infty
\end{aligned}
$$

and

$$
A_{1}=\left\{w \in L_{\mathrm{loc}}^{p}\left(R^{n}\right): M(w)(x) \leq C w(x), \text { a.e. }\right\} .
$$

The $A(p, q)$ weight is defined by [14], for $1<p, q<\infty$,

$$
A(p, q)=\left\{w>0: \sup _{Q}\left(\frac{1}{|Q|} \int_{Q} w(x)^{q} d x\right)^{1 / q}\left(\frac{1}{|Q|} \int_{Q} w(x)^{-p /(p-1)} d x\right)^{(p-1) / p}<\infty\right\} .
$$

Given a non-negative weight function $w$, for $1 \leq p<\infty$, the weighted Lebesgue space $L^{p}(w)$ is the space of functions $f$ such that

$$
\|f\|_{L^{p}(w)}=\left(\int_{R^{n}}|f(x)|^{p} w(x) d x\right)^{1 / p}<\infty .
$$

For $\beta>0, p>1$ and the non-negative weight function $w$, let $\dot{F}_{p}^{\beta, \infty}(w)$ be the weighted homogeneous Triebel-Lizorkin space [9].

For $0<\beta<1$ and the non-negative weight function $w$, the weighted Lipschitz space $\operatorname{Lip}_{\beta}(w)$ is the space of functions $b$ such that

$$
\|b\|_{\operatorname{Lip}_{\beta}(w)}=\sup _{Q} \frac{1}{w(Q)^{1+\beta / n}} \int_{Q}\left|b(y)-b_{Q}\right| d y<\infty .
$$

Remark (1) For $b \in \operatorname{Lip}_{\beta}(w), w \in A_{1}$ and $x \in Q$, it is well known that

$$
\left|b_{Q}-b_{2^{k} Q}\right| \leq C k\|b\|_{\operatorname{Lip}_{\beta}(w)} w(x) w\left(2^{k} Q\right)^{\beta / n} .
$$

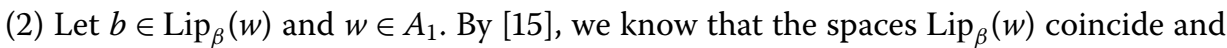
the norms $\|b\|_{\operatorname{Lip}_{\beta}(w)}$ are equivalent with respect to different values of $1 \leq p \leq \infty$.

Definition Let $T: S \rightarrow S^{\prime}$ be a bounded linear operator. $T$ is called a strongly singular integral operator if it satisfies the following conditions:

(i) $T$ extends to a bounded operator on $L^{2}\left(R^{n}\right)$; 
(ii) there exists a function $K(x, y)$ continuous away from the diagonal on $R^{n} \times R^{n}$ such that

$$
|K(x, y)-K(x, z)|+|K(y, x)-K(z, x)| \leq C|y-z|^{\delta}|x-z|^{n+\delta / \varepsilon}
$$

if $2|y-z|^{\varepsilon} \leq|x-z|$ for some $0<\delta \leq 1,0<\varepsilon<1$, and

$(T f, g)=\int_{R^{n}} \int_{R^{n}} K(x, y) f(y) g(x) d y d x$ for $f, g \in S$ with disjoint support;

(iii) for some $(1-\varepsilon) n / 2 \leq \beta<n / 2, T$ and $T^{*}$ extend to a bounded operator from $L^{q}\left(R^{n}\right)$ into $L^{2}\left(R^{n}\right)$, where $1 / q=1 / 2+\beta / n$.

Let $b$ be a locally integrable function on $R^{n}$. The Toeplitz type operator related to $T$ is defined by

$$
T_{b}=\sum_{k=1}^{m} T^{k, 1} M_{b} T^{k, 2}
$$

where $T^{k, 1}$ are strongly singular integral operators or $\pm I$ (the identity operator), $T^{k, 2}$ are bounded linear operators on $L^{p}\left(R^{n}\right)$ for $1<p<\infty, k=1, \ldots, m, M_{b}(f)=b f$.

Note that the commutator $[b, T](f)=b T(f)-T(b f)$ is a particular case of the Toeplitz type operators $T_{b}$. The Toeplitz type operators $T_{b}$ are non-trivial generalizations of the commutator. It is well known that commutators are of great interest in harmonic analysis and have been widely studied by many authors $[4,5]$. In [16-19], the boundedness of the strongly singular integral operator is obtained. In [20], a sharp function estimate of the strongly singular integral operator is obtained. In [21], the boundedness of the strongly singular integral operators and their commutators is obtained. In [13], the Toeplitz type operators related to the strongly singular integral operators are introduced, and the boundedness for the operators generated by $B M O$ and Lipschitz functions is obtained. Our works are motivated by these papers. The main purpose of this paper is to prove sharp maximal inequalities for the Toeplitz type operators $T_{b}$. As applications, we obtain the weighted $L^{p}$-norm inequality and the Triebel-Lizorkin space boundedness for the Toeplitz type operators $T_{b}$.

We need the following preliminary lemmas.

Lemma 1 ([16]) Let $T$ be a strongly singular integral operator. Then $T$ is bounded on $L^{p}(w)$ for $w \in A_{p}$ with $1<p<\infty$, and when $((1-\varepsilon) n+2 \beta) / 2 \beta<u \leq 2,0<u / v \leq \delta, T$ is bounded from $L^{u}\left(R^{n}\right)$ into $L^{v}\left(R^{n}\right)$.

Lemma 2 ([15]) For any cube $Q, b \in \operatorname{Lip}_{\beta}(w), 0<\beta<1$, and $w \in A_{1}$, we have

$$
\sup _{x \in Q}\left|b(x)-b_{Q}\right| \leq C\|b\|_{\operatorname{Lip}_{\beta}(w)} w(Q)^{1+\beta / n}|Q|^{-1} .
$$

Lemma 3 ([9]) For $0<\beta<1,1<p<\infty$, and $w \in A_{\infty}$, we have

$$
\begin{aligned}
\|f\|_{\dot{F}_{p}^{\beta, \infty}(w)} & \approx\left\|\sup _{Q \ni \cdot} \frac{1}{|Q|^{1+\beta / n}} \int_{Q}\left|f(x)-f_{Q}\right| d x\right\|_{L^{p}(w)} \\
& \approx\left\|\sup _{Q \ni \cdot c} \inf _{c} \frac{1}{|Q|^{1+\beta / n}} \int_{Q}|f(x)-c| d x\right\|_{L^{p}(w)} .
\end{aligned}
$$


Lemma 4 ([1]) Let $0<p<\infty$ and $w \in \bigcup_{1 \leq r<\infty} A_{r}$. Then, for any smooth function $f$ for which the left-hand side is finite,

$$
\int_{R^{n}} M(f)(x)^{p} w(x) d x \leq C \int_{R^{n}} M^{\#}(f)(x)^{p} w(x) d x .
$$

Lemma 5 ([14]) Suppose that $0<\eta<n, 1<s<p<n / \eta, 1 / q=1 / p-\eta / n$, and $w \in A(p, q)$. Then

$$
\left\|M_{\eta, s}(f)\right\|_{L^{q}\left(w^{q)}\right.} \leq C\|f\|_{L^{p}\left(w^{p}\right)}
$$

\section{Theorems and proofs}

We shall prove the following theorems.

Theorem 1 Let $w \in A_{1}, 0<\beta<1, b \in \operatorname{Lip}_{\beta}(w)$, and $((1-\varepsilon) n+2 \beta) / 2 \beta<s<n / \beta$. If $g \in L^{p}\left(R^{n}\right)$ $(1<p<\infty)$ and $T_{1}(g)=0$, then there exists a constant $C>0$ such that, for any $f \in C_{0}^{\infty}\left(R^{n}\right)$ and $\tilde{x} \in R^{n}$,

$$
M^{\#}\left(T_{b}(f)\right)(\tilde{x}) \leq C\|b\|_{\operatorname{Lip}_{\beta}(w)} w(\tilde{x})^{1+\beta / n} \sum_{k=1}^{m} M_{\beta, s}\left(T^{k, 2}(f)\right)(\tilde{x}) .
$$

Theorem 2 Let $w \in A_{1}, 0<\beta<\min (1, \delta / \varepsilon),((1-\varepsilon) n+2 \beta) / 2 \beta<s<n / \beta$, and $b \in \operatorname{Lip}_{\beta}(w)$. If $g \in L^{p}\left(R^{n}\right)(1<p<\infty)$ and $T_{1}(g)=0$, then there exists a constant $C>0$ such that, for any $f \in C_{0}^{\infty}\left(R^{n}\right)$ and $\tilde{x} \in R^{n}$,

$$
\sup _{Q \ni \tilde{x} \in R^{n}} \frac{1}{|Q|^{1+\beta / n}} \int_{Q}\left|T_{b}(f)(x)-c\right| d x \leq C\|b\|_{\operatorname{Lip}_{\beta}(w)} w(\tilde{x})^{1+\beta / n} \sum_{k=1}^{m} M_{s}\left(T^{k, 2}(f)\right)(\tilde{x}) .
$$

Theorem 3 Let $w \in A_{1}, 0<\beta<1,1 / q=1 / p-\beta / n$, and $b \in \operatorname{Lip}_{\beta}(w)$. If $g \in L^{p}\left(R^{n}\right)(1<p<\infty)$ and $T_{1}(g)=0$, then $T_{b}$ is bounded from $L^{p}(w)$ to $L^{q}\left(w^{q / p-q(1+\beta / n)}\right)$.

Theorem 4 Let $w \in A_{1}, 0<\beta<\min (1, \delta / \varepsilon), 1<p<n / m \beta, 1 / q=1 / p-\beta / n$, and $b \in \operatorname{Lip}_{\beta}(w)$. If $g \in L^{p}\left(R^{n}\right)(1<p<\infty)$ and $T_{1}(g)=0$, then $T_{b}$ is bounded from $L^{p}(w)$ to $\dot{F}_{q}^{\beta, \infty}\left(w^{q / p-q(1+\beta / n)}\right)$.

Proof of Theorem 1 It suffices to prove for $f \in C_{0}^{\infty}\left(R^{n}\right)$ and some constant $C_{0}$ that the following inequality holds:

$$
\frac{1}{|Q|} \int_{Q}\left|T_{b}(f)(x)-C_{0}\right| d x \leq C\|b\|_{\operatorname{Lip}_{\beta}(w)} w(\tilde{x})^{1+\beta / n} \sum_{k=1}^{m} M_{\beta, s}\left(T^{k, 2}(f)\right)(\tilde{x}) .
$$

Without loss of generality, we may assume $T^{k, 1}$ are $T(k=1, \ldots, m)$. Fix a cube $Q=Q\left(x_{0}, d\right)$ and $\tilde{x} \in Q$. We have the following two cases.

Case 1. $d>1$. Write

$$
T_{b}(f)(x)=T_{b-b_{Q}}(f)(x)=T_{\left(b-b_{Q}\right) \chi_{2 Q}}(f)(x)+T_{\left(b-b_{Q}\right) \chi_{(2 Q)}}(f)(x)=f_{1}(x)+f_{2}(x) .
$$

Then

$$
\frac{1}{|Q|} \int_{Q}\left|T_{b}(f)(x)-f_{2}\left(x_{0}\right)\right| d x \leq \frac{1}{|Q|} \int_{Q}\left|f_{1}(x)\right| d x+\frac{1}{|Q|} \int_{Q}\left|f_{2}(x)-f_{2}\left(x_{0}\right)\right| d x=I_{1}+I_{2} .
$$


Chen Journal of Inequalities and Applications 2014, 2014:42

Page 5 of 11

http://www.journalofinequalitiesandapplications.com/content/2014/1/42

For $I_{1}$, by Hölder's inequality, boundedness of $T$, and Lemma 2, we obtain

$$
\begin{aligned}
& \frac{1}{|Q|} \int_{Q}\left|T^{k, 1} M_{\left(b-b_{Q}\right) \chi_{2 Q}} T^{k, 2}(f)(x)\right| d x \\
& \leq\left(\frac{1}{|Q|} \int_{R^{n}}\left|T^{k, 1} M_{\left(b-b_{Q}\right) \chi_{2 Q}} T^{k, 2}(f)(x)\right|^{s} d x\right)^{1 / s} \\
& \leq C|Q|^{-1 / s}\left(\int_{2 Q}\left|M_{\left(b-b_{Q}\right) \chi_{2 Q}} T^{k, 2}(f)(x)\right|^{s} d x\right)^{1 / s} \\
& \leq C|Q|^{-1 / s}\left(\int_{2 Q}\left(\left|b(x)-b_{Q}\right|\left|T^{k, 2}(f)(x)\right|\right)^{s} d x\right)^{1 / s} \\
& \leq C|Q|^{-1 / s} \sup _{x \in 2 Q}\left|b(x)-b_{2 Q}\right|\left(\int_{Q}\left|T^{k, 2}(f)(x)\right|^{s} d x\right)^{1 / s} \\
& \leq C|Q|^{-1 / s}\|b\|_{\operatorname{Lip}_{\beta}(w)} \frac{w(2 Q)^{1+\beta / n}}{|2 Q|}|Q|^{1 / s-\beta / n}\left(\frac{1}{|Q|^{1-s \beta / n}} \int_{Q}\left|T^{k, 2}(f)(x)\right|^{s} d x\right)^{1 / s} \\
& \leq C\|b\|_{\operatorname{Lip}_{\beta}(w)}\left(\frac{w(Q)}{|Q|}\right)^{1+\beta / n} M_{\beta, s}\left(T^{k, 2}(f)\right)(\tilde{x}) \\
& \leq C\|b\|_{\operatorname{Lip}_{\beta}(w)} w(\tilde{x})^{1+\beta / n} M_{\beta, s}\left(T^{k, 2}(f)\right)(\tilde{x}),
\end{aligned}
$$

thus

$$
\begin{aligned}
I_{1} & \leq \sum_{k=1}^{m} \frac{1}{|Q|} \int_{Q}\left|T^{k, 1} M_{\left(b-b_{Q}\right) \chi_{2 Q}} T^{k, 2}(f)(x)\right| d x \\
& \leq C\|b\|_{\operatorname{Lip}_{\beta}} w(\tilde{x})^{1+\beta / n} \sum_{k=1}^{m} M_{\beta, s}\left(T^{k, 2}(f)\right)(\tilde{x}) .
\end{aligned}
$$

For $I_{2}$, by $d>1$ and $2\left|x-x_{0}\right|^{\varepsilon} \leq\left|y-x_{0}\right|$ for $x \in Q$ and $y \in(2 Q)^{c}$, we obtain, for $x \in Q$,

$$
\begin{aligned}
&\left|T^{k, 1} M_{\left(b-b_{Q}\right) x_{(2 Q)}{ }^{c}} T^{k, 2}(f)(x)-T^{k, 1} M_{\left(b-b_{Q}\right) \chi_{(2 Q) c^{c}}} T^{k, 2}(f)\left(x_{0}\right)\right| \\
& \leq \int_{(2 Q)^{c}}\left|b(y)-b_{2 Q}\right|\left|K(x, y)-K\left(x_{0}, y\right)\right|\left|T^{k, 2}(f)(y)\right| d y \\
& \leq C \int_{(2 Q)^{c}}\left|b(y)-b_{2 Q}\right| \frac{\left|x_{0}-x\right|^{\delta}}{\left|x_{0}-y\right|^{n+\delta / \varepsilon}}\left|T^{k, 2}(f)(y)\right| d y \\
& \leq C d^{\delta} \sum_{j=1}^{\infty} \int_{2^{j} d \leq\left|y-x_{0}\right|<2^{j+1} d}\left(2^{j} d\right)^{-n-\delta / \varepsilon}\left|b(y)-b_{2^{i+1} Q}\right|\left|T^{k, 2}(f)(y)\right| d y \\
& \quad+C d^{\delta} \sum_{j=1}^{\infty}\left(2^{j} d\right)^{-n-\delta / \varepsilon}\left|b_{2^{j+1} Q}-b_{2 Q}\right| \int_{2^{j} d \leq\left|y-x_{0}\right|<2^{j+1} d}\left|T^{k, 2}(f)(y)\right| d y \\
& \leq C\|b\|_{\operatorname{Lip}_{\beta}(w)} d^{\delta-\delta / \varepsilon} \\
& \times \sum_{j=1}^{\infty} 2^{-j \delta / \varepsilon}\left(\frac{w\left(2^{j+1} Q\right)}{\left|2^{j+1} Q\right|}\right)^{1+\beta / n}\left(\frac{1}{\left|2^{j+1} Q\right|^{1-s \beta / n}} \int_{2^{j+1} Q}\left|T^{k, 2}(f)(y)\right|^{s} d y\right)^{1 / s} \\
&+C\|b\|_{\operatorname{Lip}_{\beta}(w)} d^{\delta-\delta / \varepsilon}
\end{aligned}
$$




$$
\begin{aligned}
& \times \sum_{j=1}^{\infty} j 2^{-j \delta / \varepsilon} w(\tilde{x})\left(\frac{w\left(2^{j+1} Q\right)}{\left|2^{j+1} Q\right|}\right)^{\beta / n}\left(\frac{1}{\left|2^{j+1} Q\right|^{1-s \beta / n}} \int_{2^{j+1} Q}\left|T^{k, 2}(f)(y)\right|^{s} d y\right)^{1 / s} \\
\leq & C\|b\|_{\operatorname{Lip}_{\beta}(w)} w(\tilde{x})^{1+\beta / n} M_{\beta, s}\left(T^{k, 2}(f)\right)(\tilde{x}),
\end{aligned}
$$

thus

$$
\begin{aligned}
I_{2} & \leq \frac{1}{|Q|} \int_{Q} \sum_{k=1}^{m}\left|T^{k, 1} M_{\left(b-b_{Q}\right) x_{(2 Q)}} T^{k, 2}(f)(x)-T^{k, 1} M_{\left(b-b_{Q}\right) x_{(2 Q)}} T^{k, 2}(f)\left(x_{0}\right)\right| d x \\
& \leq C\|b\|_{\operatorname{Lip}_{\beta}(w)} w(\tilde{x})^{1+\beta / n} \sum_{k=1}^{m} M_{\beta, s}\left(T^{k, 2}(f)\right)(\tilde{x}) .
\end{aligned}
$$

Case 2. $d \leq 1$. Set $\tilde{Q}=Q\left(x_{0}, d^{\varepsilon}\right)$ and write

$$
T_{b}(f)(x)=T_{b-b_{Q}}(f)(x)=T_{\left(b-b_{Q}\right) \chi_{2 \tilde{Q}}}(f)(x)+T_{\left(b-b_{Q}\right) x_{(2 \tilde{Q})}}(f)(x)=f_{1}(x)+f_{2}(x) .
$$

Then

$$
\frac{1}{|Q|} \int_{Q}\left|T_{b}(f)(x)-f_{2}\left(x_{0}\right)\right| d x \leq \frac{1}{|Q|} \int_{Q}\left|f_{1}(x)\right| d x+\frac{1}{|Q|} \int_{Q}\left|f_{2}(x)-f_{2}\left(x_{0}\right)\right| d x=I_{3}+I_{4} .
$$

For $I_{3}$, since $((1-\varepsilon) n+2 \beta) / 2 \beta \leq s<\infty$, there exists $q$ such that $r<s, 0<r / q \leq \varepsilon$, and $T$ is bounded from $L^{r}\left(R^{n}\right)$ into $L^{q}\left(R^{n}\right)$. By using the same argument as in the proof of $I_{1}$, we get

$$
\begin{aligned}
& \frac{1}{|Q|} \int_{Q}\left|T^{k, 1} M_{\left(b-b_{Q}\right) x_{2 \tilde{Q}}} T^{k, 2}(f)(x)\right| d x \\
& \quad \leq\left(\frac{1}{|Q|} \int_{R^{n}}\left|T^{k, 1} M_{\left(b-b_{Q}\right) x_{2 \tilde{Q}}} T^{k, 2}(f)(x)\right|^{q} d x\right)^{1 / q} \\
& \quad \leq C|Q|^{-1 / q}\left(\int_{R^{n}}\left|\left(b(x)-b_{2 Q}\right) f_{1}(x)\right|^{r} d x\right)^{1 / r} \\
& \quad \leq C|Q|^{-1 / q}\left(\int_{2 \tilde{Q}}\left(\left|b(x)-b_{2 \tilde{Q}}\right|^{r}+\left|b_{2 \tilde{Q}}-b_{2 Q}\right|^{r}\right)\left|T^{k, 2}(f)(x)\right|^{r} d x\right)^{1 / r} \\
& \quad \leq C\|b\|_{\operatorname{Lip}_{\beta}(w)}|Q|^{-1 / q}\left(w(\tilde{Q})^{1+\beta / n}|\tilde{Q}|^{-1}+w(\tilde{x}) w(\tilde{Q})^{\beta / n}\right)\left(\int_{2 \tilde{Q}}\left|T^{k, 2}(f)(x)\right|^{r} d x\right)^{1 / r} \\
& \leq C\|b\|_{\operatorname{Lip}_{\beta}(w)} d^{n(\varepsilon / r-1 / q)} w(\tilde{x})\left(\frac{w(\tilde{Q})}{|\tilde{Q}|}\right)^{\beta / n}\left(\frac{1}{|\tilde{Q}|^{1-s \beta / n}} \int_{\tilde{Q}}\left|T^{k, 2}(f)(x)\right|^{s} d x\right)^{1 / s} \\
& \quad \leq C\|b\|_{\operatorname{Lip}_{\beta}(w)} w(\tilde{x})^{1+\beta / n} M_{\beta, s}\left(T^{k, 2}(f)\right)(\tilde{x}),
\end{aligned}
$$

thus

$$
\begin{aligned}
I_{3} & \leq \sum_{k=1}^{m} \frac{1}{|Q|} \int_{Q}\left|T^{k, 1} M_{\left(b-b_{Q}\right) \chi_{2} \tilde{Q}} T^{k, 2}(f)(x)\right| d x \\
& \leq C\|b\|_{\operatorname{Lip}_{\beta} w(\tilde{x})^{1+\beta / n}} \sum_{k=1}^{m} M_{\beta, s}\left(T^{k, 2}(f)\right)(\tilde{x}) .
\end{aligned}
$$


For $I_{4}$, by using the same argument as in the proof of $I_{2}$, we get, for $x \in Q$,

$$
\begin{aligned}
& \left|T^{k, 1} M_{\left(b-b_{Q}\right) \chi_{(2 \tilde{Q})^{c}}} T^{k, 2}(f)(x)-T^{k, 1} M_{\left(b-b_{Q}\right) \chi_{(2 \tilde{Q})^{c}}} T^{k, 2}(f)\left(x_{0}\right)\right| \\
& \leq \int_{(2 \tilde{Q})^{c}}\left|b(y)-b_{2 Q}\right|\left|K(x, y)-K\left(x_{0}, y\right)\right|\left|T^{k, 2}(f)(y)\right| d y \\
& \leq C \int_{(2 \tilde{Q})^{c}}\left|b(y)-b_{2 Q}\right||f(y)| \frac{\left|x_{0}-x\right|^{\delta}}{\left|x_{0}-y\right|^{n+\delta / \varepsilon}} d y \\
& \leq C d^{\delta} \sum_{j=1}^{\infty}\left(2^{j} d^{\varepsilon}\right)^{-n-\delta / \varepsilon} \int_{2^{j+1} \tilde{Q}}\left|b(y)-b_{2^{j+1}} \tilde{Q}\right|\left|T^{k, 2}(f)(y)\right| d y \\
& +C d^{\delta} \sum_{j=1}^{\infty}\left(2^{j} d^{\varepsilon}\right)^{-n-\delta / \varepsilon}\left|b_{2^{j+1} \tilde{Q}}-b_{2 \tilde{Q}}\right| \int_{2^{j+1} \tilde{Q}}\left|T^{k, 2}(f)(y)\right| d y \\
& +C d^{\delta} \sum_{j=1}^{\infty}\left(2^{j} d^{\varepsilon}\right)^{-n-\delta / \varepsilon}\left|b_{2 \tilde{Q}}-b_{2 Q}\right| \int_{2^{j+1} \tilde{Q}}\left|T^{k, 2}(f)(y)\right| d y \\
& \leq C\|b\|_{\operatorname{Lip}_{\beta}(w)} \sum_{j=1}^{\infty} 2^{-j \delta / \varepsilon}\left(\frac{w\left(2^{j+1} \tilde{Q}\right)}{\left|2^{j+1} \tilde{Q}\right|}\right)^{1+\beta / n}\left(\frac{1}{\left|2^{j+1} \tilde{Q}\right|^{1-s \beta / n}} \int_{2^{j+1} Q}\left|T^{k, 2}(f)(y)\right|^{s} d y\right)^{1 / s} \\
& +C\|b\|_{\operatorname{Lip}_{\beta}(w)} \\
& \times \sum_{j=1}^{\infty} j 2^{-j \delta / \varepsilon} w(\tilde{x})\left(\frac{w\left(2^{j+1} \tilde{Q}\right)}{\left|2^{j+1} \tilde{Q}\right|}\right)^{\beta / n}\left(\frac{1}{\left|2^{j+1} \tilde{Q}\right|^{1-s \beta / n}} \int_{2^{j+1} \tilde{Q}}\left|T^{k, 2}(f)(y)\right|^{s} d y\right)^{1 / s} \\
& +C\|b\|_{\operatorname{Lip}_{\beta}(w)} \sum_{j=1}^{\infty} 2^{-j \delta / \varepsilon} w(\tilde{x})\left(\frac{w(\tilde{Q})}{|\tilde{Q}|}\right)^{\beta / n}\left(\frac{1}{\left|2^{j+1} \tilde{Q}\right|^{1-s \beta / n}} \int_{2^{j+1} \tilde{Q}}\left|T^{k, 2}(f)(y)\right|^{s} d y\right)^{1 / s} \\
& \leq C\|b\|_{\operatorname{Lip}_{\beta}(w)} w(\tilde{x})^{1+\beta / n} M_{\beta, s}\left(T^{k, 2}(f)\right)(\tilde{x}),
\end{aligned}
$$

thus

$$
\begin{aligned}
I_{4} & \leq \frac{1}{|Q|} \int_{Q} \sum_{k=1}^{m}\left|T^{k, 1} M_{\left(b-b_{Q}\right) \chi_{(2 Q)}{ }^{c}} T^{k, 2}(f)(x)-T^{k, 1} M_{\left(b-b_{Q}\right) \chi_{(2 Q)}} T^{k, 2}(f)\left(x_{0}\right)\right| d x \\
& \leq C\|b\|_{\operatorname{Lip}_{\beta}(w)} w(\tilde{x})^{1+\beta / n} \sum_{k=1}^{m} M_{\beta, s}\left(T^{k, 2}(f)\right)(\tilde{x}) .
\end{aligned}
$$

These complete the proof of Theorem 1 .

Proof of Theorem 2 It suffices to prove for $f \in C_{0}^{\infty}\left(R^{n}\right)$ and some constant $C_{0}$ that the following inequality holds:

$$
\frac{1}{|Q|^{1+\beta / n}} \int_{Q}\left|T_{b}(f)(x)-C_{0}\right| d x \leq C\|b\|_{\operatorname{Lip}_{\beta}(w)} w(\tilde{x})^{1+\beta / n} \sum_{k=1}^{m} M_{s}\left(T^{k, 2}(f)\right)(\tilde{x}) .
$$

Without loss of generality, we may assume $T^{k, 1}$ are $T(k=1, \ldots, m)$. Fix a cube $Q=Q\left(x_{0}, d\right)$ and $\tilde{x} \in Q$. We have the following two cases. 
Case 1. $d>1$. Similar to the proof of Theorem 1, we have

$$
T_{b}(f)(x)=T_{b-b_{Q}}(f)(x)=T_{\left(b-b_{Q}\right) \chi_{2 Q}}(f)(x)+T_{\left(b-b_{Q}\right) \chi_{(2 Q)}}(f)(x)=f_{1}(x)+f_{2}(x)
$$

and

$$
\begin{aligned}
& \frac{1}{|Q|^{1+\beta / n}} \int_{Q}\left|T_{b}(f)(x)-f_{2}\left(x_{0}\right)\right| d x \\
& \leq \frac{1}{|Q|^{1+\beta / n}} \int_{Q}\left|f_{1}(x)\right| d x+\frac{1}{|Q|^{1+\beta / n}} \int_{Q}\left|f_{2}(x)-f_{2}\left(x_{0}\right)\right| d x=I I_{1}+I I_{2} .
\end{aligned}
$$

By using the same argument as in the proof of Theorem 1, we get

$$
\begin{aligned}
& I I_{1} \leq \sum_{k=1}^{m} \frac{C}{|Q|^{\beta / n}} \sup _{x \in 2 Q}\left|b(x)-b_{2 Q}\right||Q|^{-1 / s}\left(\int_{2 Q}\left|T^{k, 2}(f)(x)\right|^{s} d x\right)^{1 / s} \\
& \leq \sum_{k=1}^{m}\|b\|_{\operatorname{Lip}_{\beta}(w)}\left(\frac{w(Q)}{|Q|}\right)^{1+\beta / n}\left(\frac{1}{|2 Q|} \int_{2 Q}\left|T^{k, 2}(f)(x)\right|^{s} d x\right)^{1 / s} \\
& \leq C\|b\|_{\operatorname{Lip}_{\beta}(w)} w(\tilde{x})^{1+\beta / n} \sum_{k=1}^{m} M_{s}\left(T^{k, 2}(f)\right)(\tilde{x}), \\
& I I_{2} \leq \frac{1}{|Q|^{1+\beta / n}} \int_{Q} \int_{(2 Q)^{c}}\left|b(y)-b_{2 Q}\right|\left|K(x, y)-K\left(x_{0}, y\right)\right|\left|T^{k, 2}(f)(y)\right| d y d x \\
& \leq \frac{C}{|Q|^{1+\beta / n}} \int_{Q} \int_{(2 Q)^{c}}\left|b(y)-b_{2 Q}\right| \frac{\left|x_{0}-x\right|^{\delta}}{\left|x_{0}-y\right|^{n+\delta / \varepsilon}}\left|T^{k, 2}(f)(y)\right| d y d x \\
& \leq C d^{\delta} \sum_{j=1}^{\infty}\left(2^{j} d\right)^{-n-\delta / \varepsilon} \int_{2^{j+1} Q}\left|b(y)-b_{2^{j+1} Q}\right|\left|T^{k, 2}(f)(y)\right| d y \\
& +C d^{\delta} \sum_{j=1}^{\infty}\left(2^{j} d\right)^{-n-\delta / \varepsilon}\left|b_{2^{j+1} Q}-b_{2 Q}\right| \int_{2^{j+1} Q}\left|T^{k, 2}(f)(y)\right| d y \\
& \leq C\|b\|_{\operatorname{Lip}_{\beta}(w)} d^{\delta-\delta / \varepsilon} \\
& \times \sum_{j=1}^{\infty} 2^{j(\beta-\delta / \varepsilon)}\left(\frac{w\left(2^{j+1} Q\right)}{\left|2^{j+1} Q\right|}\right)^{1+\beta / n}\left(\frac{1}{\left|2^{j+1} Q\right|} \int_{2^{j+1} Q}\left|T^{k, 2}(f)(y)\right|^{s} d y\right)^{1 / s} \\
& +C\|b\|_{\operatorname{Lip}_{\beta}(w)} d^{\delta-\delta / \varepsilon} \\
& \times \sum_{j=1}^{\infty} j 2^{j(\beta-\delta / \varepsilon)} w(\tilde{x})\left(\frac{w\left(2^{j+1} Q\right)}{\left|2^{j+1} Q\right|}\right)^{\beta / n}\left(\frac{1}{\left|2^{j+1} Q\right|} \int_{2^{j+1} Q}\left|T^{k, 2}(f)(y)\right|^{s} d y\right)^{1 / s} \\
& \leq C\|b\|_{\operatorname{Lip}_{\beta}(w)} w(\tilde{x})^{1+\beta / n} \sum_{k=1}^{m} M_{s}\left(T^{k, 2}(f)\right)(\tilde{x}) .
\end{aligned}
$$

Case 2. $d \leq 1$. Set $\tilde{Q}=Q\left(x_{0}, d^{\rho}\right)$, where $\rho=(\delta-\beta) /(\delta / \varepsilon-\beta)<\varepsilon$, and write

$$
T_{b}(f)(x)=T_{b-b_{Q}}(f)(x)=T_{\left(b-b_{Q}\right) \chi_{2 \tilde{Q}}}(f)(x)+T_{\left(b-b_{Q}\right) \chi_{(2 \tilde{Q})^{c}}}(f)(x)=f_{1}(x)+f_{2}(x)
$$


and

$$
\begin{aligned}
& \frac{1}{|Q|^{1+\beta / n}} \int_{Q}\left|T_{b}(f)(x)-f_{2}\left(x_{0}\right)\right| d x \\
& \leq \frac{1}{|Q|^{1+\beta / n}} \int_{Q}\left|f_{1}(x)\right| d x+\frac{1}{|Q|^{1+\beta / n}} \int_{Q}\left|f_{2}(x)-f_{2}\left(x_{0}\right)\right| d x=I I_{3}+I I_{4} .
\end{aligned}
$$

By using the same argument as in the proof of Theorem 1 , for $((1-\varepsilon) n+2 \beta) / 2 \beta \leq s<\infty$, there exists $q$ such that $r<s, 0<r / q \leq \varepsilon$, and $T$ is bounded from $L^{r}\left(R^{n}\right)$ into $L^{q}\left(R^{n}\right)$, and we get

$$
\begin{aligned}
& I I_{3} \leq \sum_{k=1}^{m} \frac{1}{|Q|} \int_{Q}\left|T^{k, 1} M_{\left(b-b_{Q}\right) \chi_{2} \tilde{Q}} T^{k, 2}(f)(x)\right| d x \\
& \leq \sum_{k=1}^{m} \frac{1}{|Q|^{\beta / n}}\left(\frac{1}{|Q|} \int_{R^{n}}\left|T^{k, 1} M_{\left(b-b_{Q}\right) \chi_{2 \tilde{Q}}} T^{k, 2}(f)(x)\right|^{q} d x\right)^{1 / q} \\
& \leq C \sum_{k=1}^{m} d^{-\beta-n / q}\left(\int_{R^{n}}\left|\left(b(x)-b_{2 Q}\right) f_{1}(x)\right|^{r} d x\right)^{1 / r} \\
& \leq C \sum_{k=1}^{m} d^{-\beta-n / q}\left(\int_{2 \tilde{Q}}\left(\left|b(x)-b_{2 \tilde{Q}}\right|^{r}+\left|b_{2 \tilde{Q}}-b_{2 Q}\right|^{r}\right)\left|T^{k, 2}(f)(x)\right|^{r} d x\right)^{1 / r} \\
& \leq C \sum_{k=1}^{m}\|b\|_{\operatorname{Lip}_{\beta}(w)} d^{-\beta-n / q}\left(w(\tilde{Q})^{1+\beta / n}|\tilde{Q}|^{-1}+w(\tilde{x}) w(\tilde{Q})^{\beta / n}\right)\left(\int_{2 \tilde{Q}}\left|T^{k, 2}(f)(x)\right|^{r} d x\right)^{1 / r} \\
& \leq C \sum_{k=1}^{m}\|b\|_{\operatorname{Lip}_{\beta}(w)} d^{\rho(n / s+\beta)-\beta-n / q} w(\tilde{x})\left(\frac{w(\tilde{Q})}{|\tilde{Q}|}\right)^{\beta / n}\left(\frac{1}{|\tilde{Q}|} \int_{\tilde{Q}}\left|T^{k, 2}(f)(x)\right|^{s} d x\right)^{1 / s} \\
& \leq C\|b\|_{\operatorname{Lip}_{\beta}(w)} w(\tilde{x})^{1+\beta / n} \sum_{k=1}^{m} M_{s}\left(T^{k, 2}(f)\right)(\tilde{x}), \\
& I I_{4} \leq \sum_{k=1}^{m} \frac{1}{|Q|^{1+\beta / n}} \int_{Q} \int_{(2 \tilde{Q})^{c}}\left|b(y)-b_{2 Q}\right|\left|K(x, y)-K\left(x_{0}, y\right)\right|\left|T^{k, 2}(f)(y)\right| d y d x \\
& \leq \sum_{k=1}^{m} \frac{C}{|Q|^{1+\beta / n}} \int_{Q} \int_{(2 \tilde{Q})^{c}}\left|b(y)-b_{2 Q}\right| \frac{\left|x_{0}-x\right|^{\delta}}{\left|x_{0}-y\right|^{n+\delta / \varepsilon}}\left|T^{k, 2}(f)(y)\right| d y d x \\
& \leq C \sum_{k=1}^{m} d^{\delta-\beta} \sum_{j=1}^{\infty}\left(2^{j} d^{\rho}\right)^{-n-\delta / \varepsilon} \int_{2^{j+1} \tilde{Q}}\left|b(y)-b_{2^{j+1}} \tilde{Q}\right|\left|T^{k, 2}(f)(y)\right| d y \\
& +C \sum_{k=1}^{m} d^{\delta-\beta} \sum_{j=1}^{\infty}\left(2^{j} d^{\rho}\right)^{-n-\delta / \varepsilon}\left|b_{2^{j+1} \tilde{Q}}-b_{2 \tilde{Q}}\right| \int_{2^{j+1} \tilde{Q}}\left|T^{k, 2}(f)(y)\right| d y \\
& +C \sum_{k=1}^{m} d^{\delta-\delta} \sum_{j=1}^{\infty}\left(2^{j} d^{\rho}\right)^{-n-\delta / \varepsilon}\left|b_{2 \tilde{Q}}-b_{2 Q}\right| \int_{2^{j+1} \tilde{Q}}\left|T^{k, 2}(f)(y)\right| d y \\
& \leq C \sum_{k=1}^{m}\|b\|_{\operatorname{Lip}_{\beta}(w)} \sum_{j=1}^{\infty} 2^{j(\beta-\delta / \varepsilon)}\left(\frac{w\left(2^{j+1} \tilde{Q}\right)}{\left|2^{j+1} \tilde{Q}\right|}\right)^{1+\beta / n}\left(\frac{1}{\left|2^{j+1} \tilde{Q}\right|} \int_{2^{s+1} Q}\left|T^{k, 2}(f)(y)\right|^{s} d y\right)^{1 / s} \\
& +C \sum_{k=1}^{m}\|b\|_{\operatorname{Lip}_{\beta}(w)}
\end{aligned}
$$




$$
\begin{aligned}
& \quad \times \sum_{j=1}^{\infty} j 2^{j(\beta-\delta / \varepsilon)} w(\tilde{x})\left(\frac{w\left(2^{j+1} \tilde{Q}\right)}{\left|2^{j+1} \tilde{Q}\right|}\right)^{\beta / n}\left(\frac{1}{\left|2^{j+1} \tilde{Q}\right|} \int_{2^{j+1} \tilde{Q}}\left|T^{k, 2}(f)(y)\right|^{s} d y\right)^{1 / s} \\
& +C \sum_{k=1}^{m}\|b\|_{\operatorname{Lip}_{\beta}(w)} \sum_{j=1}^{\infty} j 2^{j(\beta-\delta / \varepsilon)} w(\tilde{x})\left(\frac{w(\tilde{Q})}{|\tilde{Q}|}\right)^{\beta / n}\left(\frac{1}{\left|2^{j+1} \tilde{Q}\right|} \int_{2^{j+1} \tilde{Q}}\left|T^{k, 2}(f)(y)\right|^{s} d y\right)^{1 / s} \\
& \leq C\|b\|_{\operatorname{Lip}_{\beta}(w)} w(\tilde{x})^{1+\beta / n} \sum_{k=1}^{m} M_{s}\left(T^{k, 2}(f)\right)(\tilde{x}) .
\end{aligned}
$$

This completes the proof of Theorem 2 .

Proof of Theorem 3 Choose $1<s<p$ in Theorem 1, notice that $w^{q / p-q(1+\beta / n)} \in A_{\infty}$ and $w^{1 / p} \in A(p, q)$, and we have, by Lemmas 1,4 , and 5 ,

$$
\begin{aligned}
& \left\|T_{b}(f)\right\|_{\left.L^{q\left(w^{q / p}-q(1+\beta / n)\right.}\right)} \leq\left\|M\left(T_{b}(f)\right)\right\|_{\left.L^{q\left(w^{q / p}-q(1+\beta / n)\right.}\right)} \\
& \leq C\left\|M^{\#}\left(T_{b}(f)\right)\right\|_{L^{q}\left(w^{q / p-q(1+\beta / n)}\right)}
\end{aligned}
$$

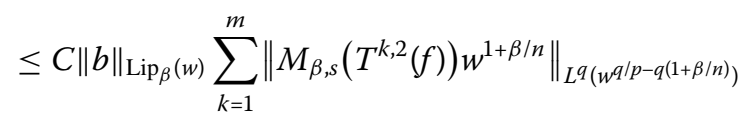

$$
\begin{aligned}
& =C\|b\|_{\operatorname{Lip}_{\beta}(w)} \sum_{k=1}^{m}\left\|M_{\beta, s}\left(T^{k, 2}(f)\right)\right\|_{L^{q}\left(w^{q / p}\right)} \\
& \leq C\|b\|_{\operatorname{Lip}_{\beta}(w)} \sum_{k=1}^{m}\left\|T^{k, 2}(f)\right\|_{L^{p}(w)} \\
& \leq C\|b\|_{\operatorname{Lip}_{\beta}(w)}\|f\|_{L^{p}(w)} .
\end{aligned}
$$

This completes the proof of Theorem 3 .

Proof of Theorem 4 Choose $1<s<p$ in Theorem 2. By using Lemma 3, we obtain

$$
\begin{aligned}
& \left.\left\|T_{b}(f)\right\|_{\dot{F}_{q}^{\beta, \infty}(w q / p-q(1+\beta / n)}\right) \\
& \leq C\|b\|_{\operatorname{Lip}_{\beta}(w)} \sum_{k=1}^{m}\left\|M_{s}\left(T^{k, 2}(f)\right) w^{1+\beta / n}\right\|_{L^{q}\left(w^{q / p-q(1+\beta / n)}\right)} \\
& \quad=C\|b\|_{\operatorname{Lip}_{\beta}(w)} \sum_{k=1}^{m}\left\|M_{s}\left(T^{k, 2}(f)\right)\right\|_{L^{q}\left(w^{q / p}\right)} \\
& \quad \leq C\|b\|_{\operatorname{Lip}_{\beta}(w)} \sum_{k=1}^{m}\left\|T^{k, 2}(f)\right\|_{L^{p}(w)} \\
& \leq C\|b\|_{\operatorname{Lip}_{\beta}(w)}\|f\|_{L^{p}(w) .}
\end{aligned}
$$

This completes the proof of the theorem.

Remark A typical example of strongly singular integral operators is a class of multiplier operators whose symbol is given by $\exp \left(i|\xi|^{\varepsilon}\right) /|\xi|^{\delta}$ for $0<\varepsilon<1$ and $\delta>0[18-20,22]$. 


\section{Competing interests}

The author declares that they have no competing interests.

Received: 1 August 2013 Accepted: 22 December 2013 Published: 24 Jan 2014

\section{References}

1. Garcia-Cuerva, J, Rubio de Francia, JL: Weighted Norm Inequalities and Related Topics. North-Holland Math., vol. 16. North-Holland, Amsterdam (1985)

2. Stein, EM: Harmonic Analysis: Real Variable Methods, Orthogonality and Oscillatory Integrals. Princeton University Press, Princeton (1993)

3. Coifman, RR, Rochberg, R, Weiss, G: Factorization theorems for Hardy spaces in several variables. Ann. Math. 103, 611-635 (1976)

4. Pérez, C: Endpoint estimate for commutators of singular integral operators. J. Funct. Anal. 128, 163-185 (1995)

5. Pérez, C, Trujillo-Gonzalez, R: Sharp weighted estimates for multilinear commutators. J. Lond. Math. Soc. 65, 672-692 (2002)

6. Chanillo, S: A note on commutators. Indiana Univ. Math. J. 31, 7-16 (1982)

7. Chen, WG: Besov estimates for a class of multilinear singular integrals. Acta Math. Sin. 16, 613-626 (2000)

8. Janson, S: Mean oscillation and commutators of singular integral operators. Ark. Mat. 16, 263-270 (1978)

9. Paluszynski, M: Characterization of the Besov spaces via the commutator operator of Coifman, Rochberg and Weiss Indiana Univ. Math. J. 44, 1-17 (1995)

10. Bloom, S: A commutator theorem and weighted BMO. Trans. Am. Math. Soc. 292, 103-122 (1985)

11. $\mathrm{Hu}, \mathrm{B}, \mathrm{Gu}, \mathrm{J}$ : Necessary and sufficient conditions for boundedness of some commutators with weighted Lipschitz spaces. J. Math. Anal. Appl. 340, 598-605 (2008)

12. Krantz, S, Li, S: Boundedness and compactness of integral operators on spaces of homogeneous type and applications. J. Math. Anal. Appl. 258, 629-641 (2001)

13. Lin, Y, Lu, SZ: Toeplitz type operators associated to strongly singular integral operator. Sci. China Ser. A 36, 615-630 (2006)

14. Muckenhoupt, B, Wheeden, RL: Weighted norm inequalities for fractional integral. Trans. Am. Math. Soc. 192, 261-274 (1974)

15. Garcia-Cuerva, J: Weighted $H^{P}$ spaces. Diss. Math. 162 (1979)

16. Alvarez, J, Milman, M: $H^{p}$ continuity properties of Calderón-Zygmund type operators. J. Math. Anal. Appl. 118, 63-79 (1986)

17. Alvarez, J, Milman, M: Vector-valued inequalities for strongly singular Calderón-Zygmund operators. Rev. Mat. Iberoam. 2, 405-426 (1986)

18. Fefferman, C: Inequalities for strongly singular convolution operators. Acta Math. 124, 9-36 (1970)

19. Fefferman, C, Stein, EM: $H^{P}$ spaces of several variables. Acta Math. 129, 137-193 (1972)

20. Chanillo, S: Weighted norm inequalities for strongly singular convolution operators. Trans. Am. Math. Soc. 281, 77-107 (1984)

21. Garcia-Cuerva, J, Harboure, E, Segovia, C, Torrea, JL: Weighted norm inequalities for commutators of strongly singular integrals. Indiana Univ. Math. J. 40, 1397-1420 (1991)

22. Sjölin, P: An $H^{P}$ inequality for strongly singular integrals. Math. Z. 165, 231-238 (1979)

10.1186/1029-242X-2014-42

Cite this article as: Chen: Weighted boundedness for Toeplitz type operators related to strongly singular integral operators. Journal of Inequalities and Applications 2014, 2014:42

\section{Submit your manuscript to a SpringerOpen ${ }^{\circ}$ journal and benefit from:}

- Convenient online submission

- Rigorous peer review

- Immediate publication on acceptance

- Open access: articles freely available online

- High visibility within the field

- Retaining the copyright to your article 